\title{
Drink Bottle Defect Detection Based on Machine Vision Large Data Analysis
}

\author{
Yuesheng Wang, Hua $\mathrm{Li}^{\text {a }}$ \\ School of Hangzhou Dianzi University, Hangzhou 310018, China. \\ ahdu_lihua@163.com
}

\begin{abstract}
Aiming at the problem of low efficiency, low quality and uncertainty of the subjective control of the beverage bottle defect, this paper designs a kind of beverage bottle defect detection based on machine vision large data analysis and multi-sensor information fusion. System. At the same time, a large data sample base is set up in the image data of the beverage bottle product. When the quality of the new beverage bottle is detected, a plurality of features of the image of the beverage bottle are extracted by machine learning and then compared with the large data sample database to identify the possible The existence of bottlenecks, improve the quality of beverage bottles detection efficiency. Through the use of the system to detect and use the artificial test to compare the test, fully demonstrated the system in the beverage bottle flaw detection of high efficiency and high pass rate, reached the beverage bottle product testing and packaging automation requirements are very good of the application value.
\end{abstract}

Keywords: Machine vision; large data; beverage bottle; defect detection; multi-sensor.

\section{Introduction}

With the progress of society, people's living standards improve, we have the quality of consumer goods also put forward higher requirements, the quality of products has become more and more manufacturers and consumers concerned about. In recent years, mineral water, beverage milk and other bottled products consumption growth, bottled product quality testing standards need to continuously improve. For bottled beverages, the quality of its bottle packaging directly or indirectly affect the quality of its internal beverages [1]. Although the detection method based on the sensor technology to achieve the speed and quality requirements, but the manufacturing cost of testing equipment, its flexibility is relatively poor. With the continuous development of optical technology, computer science and technology, digital image processing, machine learning of large data, pattern recognition and artificial intelligence, the beverage bottle defect detection system with high efficiency, high precision and low cost based on machine vision gradually of the accepted by many manufacturers [2].

In order to improve the accuracy and stability of the results of beverage bottle defect detection, it is necessary to expand the capacity of the sample. With the development of hardware performance, the device memory and performance have been greatly improved, and has a networking function, which is based on large data analysis machine vision defect detection system provides the conditions. At present, large data and corresponding algorithms for large data machine vision learning methods have been more extensive and in-depth, but the combination of large data and machine vision applications are rare [3]. In this paper, based on the machine vision data analysis of the beverage bottle defect detection system, to improve the quality of beverage bottles detection efficiency and accuracy of practical significance.

\section{Detection system overall structure}

As shown in Figure 1, the whole system consists of five parts: the multi-sensor module, conveyor devices, removal devices, visual systems and experimental platform using API. When the fiber optic sensor is triggered, the optical fiber sensor will trigger the camera light source, the camera will capture the image information to the control side, and analysis and processing, removal of the device 
(Figure one), after the sensor is triggered, the sensor will trigger the control side of the visual system, In the infrared range sensor will trigger the digital signal after the feedback to the control side, control the rejection device to remove defective products. The camera in the vision system continuously obtains the product image information and transmits it to the control end to ensure the passing rate of the detection. The system has good stability. The infrared distance sensor real-time records and transmits the digital signal to the control end and controls the rejecting device to remove automatically. Defective products, to ensure a high degree of automation system. At the same time, the part of API image collection interface is supplemented by the collection of Internet images by the web crawler, and the experiment and research of the feature extraction are carried out. On this basis, the defect classifier is designed and integrated into the API, Thereby improving the detection efficiency.

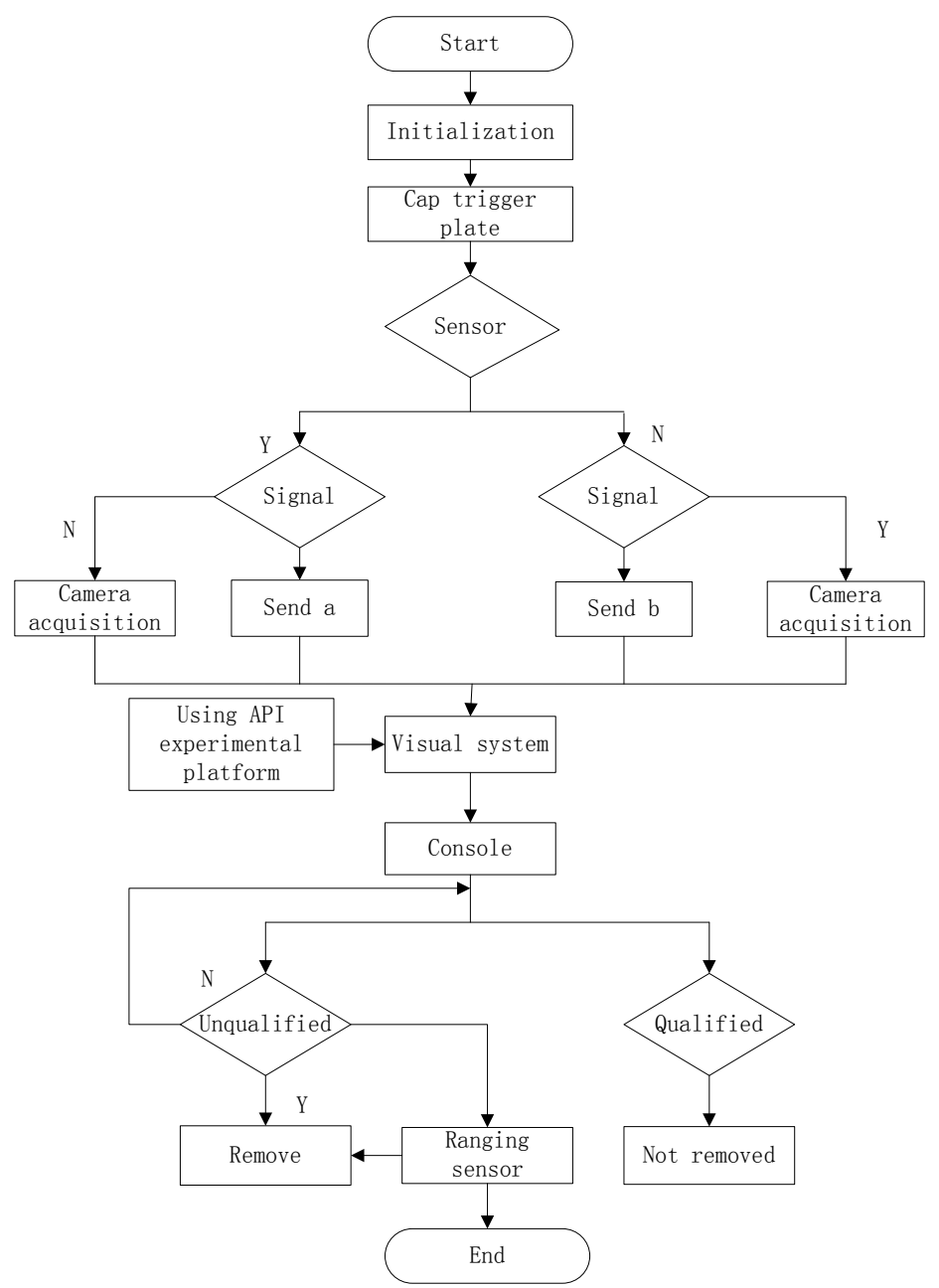

Fig. 1 The overall structure of the detection system flow chart

\section{Detection system hardware design}

\subsection{Beverage bottle cap packaging and testing equipment.}

The detection system consists of a conveyor belt device, a cap trigger plate, an adjusting frame, a rejecting device, a multi-sensor and a vision system. The cap trigger plate, the camera and the light source can be adjusted according to the size of the different beverage products, so that the system can detect a variety of different types of beverage products, so that the versatility of the system. According to the number of products to be tested on the production line, adjust the time interval of camera acquisition images to select the appropriate conveyor speed, thereby improving the detection efficiency. Multi-sensor fusion not only ensures the accuracy of the system detection, but also makes the detection more intelligent, to achieve the beverage product line detection of automation and intelligence. 


\subsection{Multi-sensor fusion.}

It is an important index of the performance of the detection system that whether the detection system can detect the unqualified products with high efficiency and high accuracy and eliminate them in the production line of the beverage bottle product defect detection. Multi-sensor fusion not only ensures the accuracy of the system detection, but also makes the detection more intelligent, to achieve the bottled beverage production line detection automation, intelligent [4].

\subsection{Beverage bottle cap image data platform construction.}

Machine vision is the basic data of the image, it has a large amount of data, unstructured features. The feature extraction method is used to extract the feature of the image, and the feature of the feature is evaluated. The principal component of the feature is selected automatically, and the feature is analyzed and studied, and the feature selection method is obtained [5]. The statistical model of the whole sample is established, and the defect classification algorithm is designed, and the algorithm is realized by the tool of parallel computation, so as to improve the operation speed of the algorithm.

Firstly, the industry standard API image collection interface part is designed, which is supplemented by the web crawler to collect the Internet image, and the relevant part of the feature extraction is experimented and studied. On this basis, the defect classifier is designed and Integrate it into the API. The establishment of experimental platform using API, the relevant experiments. Then, the data was collected while the experiment was conducted until large data capable of defect detection was formed.

Image source has two aspects, one from the testing equipment, which is the main source of image; the second is from the Internet. From the representative of the beverage industry to start with the industry associations and the corresponding large companies from the industry standard cut into the release of the corresponding API interface, the API interface integrates both the data input function, but also integrates the statistical model output interface and detection Algorithm interface, which facilitates device manufacturers to call. So that equipment manufacturers are both large data providers, but also the beneficiaries of large data. Second, to be used as a way to complement the network crawl, from the Internet to pick up the appropriate data. As the amount of data on the Internet, and the data is from various sectors of the image, you need to judge the image content, but also need to image size, format, etc. to adjust.

API to large data-based, including the feature extraction algorithm engine, statistical model interface and defect classification algorithm interface. Large data is the foundation of the research. The basic data of machine vision is image, which has the features of large data volume and unstructured. The feature extraction algorithm is used to extract the feature of the image, and the extracted data is input to the server through Ethernet. . Through the analysis and evaluation of the characteristics of the pros and cons of the principal components of the feature automatically selected to establish a statistical model of the overall sample, and the use of the minimum risk classifier algorithm.

\section{Visual inspection system design}

Detection system in the production line to detect defects in the beverage bottle to ensure that the system detects the high accuracy, trigger sensor in the bottle cap is triggered, the unqualified products directly removed device removed. In addition, in the development of good API, build an experimental platform. The vision system can be divided into the following parts: the multi-sensor module, the control end, the CCD camera and the culling device as well as the experimental platform using API. The specific structure shown in Figure 2. 


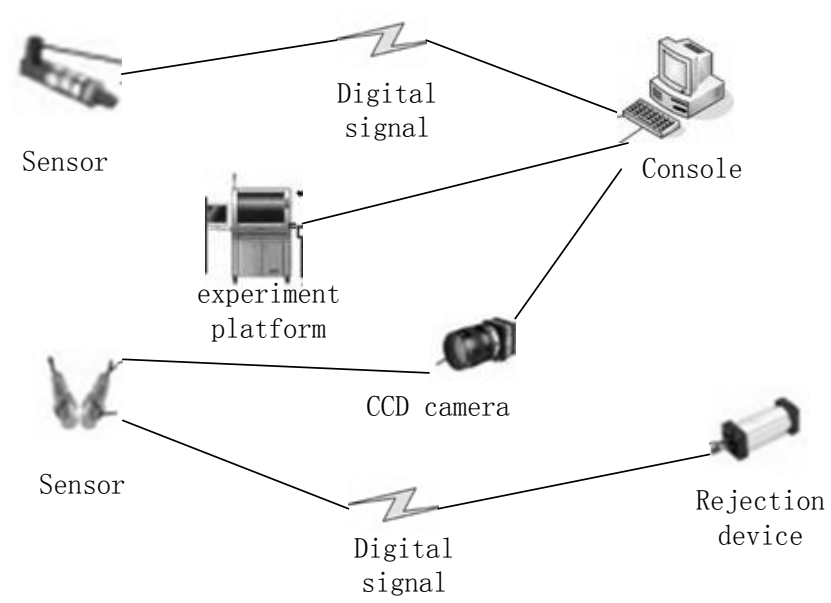

Fig. 2 Visual system structure

\section{Experiment and analysis}

System test prototype using a visual cap package detection device trigger plate sensor mounted on the trigger plate adjustment frame, CCD camera installed in the camera adjustment rack. Detection system prototype shown in Figure 3.

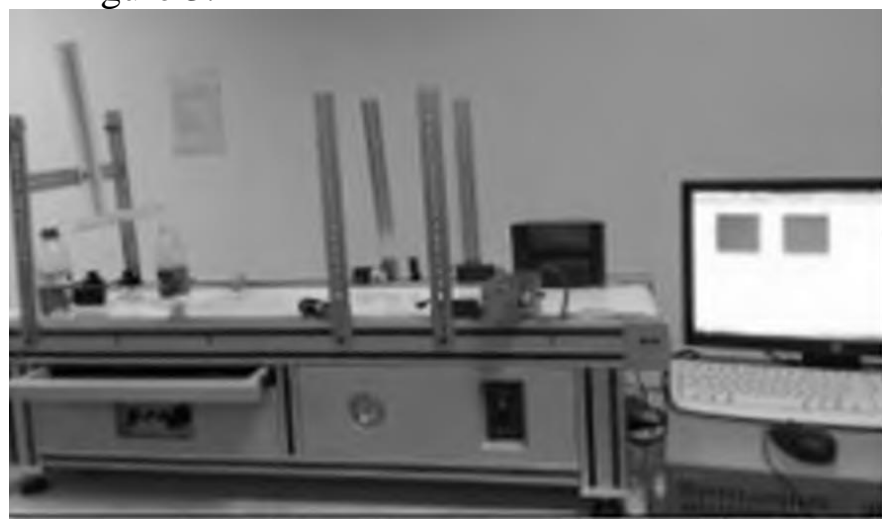

Fig. 3 Detection system prototype

\subsection{Test one.}

In the case of using only machine vision technology without large data, the test system is tested with the same number of products, and the height of different products is compared with the manual test.

Table 1 Test data

\begin{tabular}{cccccc}
\hline $\begin{array}{c}\text { Number } \\
\text { of trials }\end{array}$ & $\begin{array}{c}\text { Beverage bottle } \\
\text { product } \\
\text { height/mm }\end{array}$ & $\begin{array}{c}\text { Quantity/pi } \\
\text { ece }\end{array}$ & $\begin{array}{c}\text { System time } \\
\text { required/s }\end{array}$ & $\begin{array}{c}\text { The time required for } \\
\text { labor/s }\end{array}$ & $\begin{array}{c}\text { Time } \\
\text { difference/s }\end{array}$ \\
\hline 1 & & & 74 & 252 & 178 \\
2 & 140 & 1000 & 68 & 270 & 202 \\
3 & & & 76 & 278 & 202 \\
1 & \multirow{2}{*}{180} & 1000 & 76 & 246 & 176 \\
2 & & & 74 & 262 & 186 \\
3 & & 60 & 268 & 194 \\
1 & \multirow{2}{*}{200} & 1000 & 68 & 242 & 182 \\
2 & & & 64 & 250 & 182 \\
3 & & & 258 & 194 \\
\hline
\end{tabular}

From the test results can be seen, the detection system in the detection of the same height of the beverage bottle products, the detection system time than the manual detection of the same number of products with a short time difference of 3-3.5 minutes, the detection system with high efficiency. 


\subsection{Test two.}

In the case of using machine vision technology combined with large data, experiments were conducted by using API experimental platform. The test results were compared with those of manual inspection in the case of the same product number and different product height.

\begin{tabular}{cccccc}
\hline $\begin{array}{c}\text { Numbe } \\
\text { of trials }\end{array}$ & $\begin{array}{c}\text { Beverage bottle } \\
\text { product height/mm }\end{array}$ & $\begin{array}{c}\text { Quantity } \\
\text { /piece }\end{array}$ & $\begin{array}{c}\text { System time } \\
\text { required/s }\end{array}$ & $\begin{array}{c}\text { The time required for } \\
\text { labor/s }\end{array}$ & $\begin{array}{c}\text { Time } \\
\text { difference/s }\end{array}$ \\
\hline 1 & & & 70 & 248 & 178 \\
2 & 140 & 1000 & 64 & 275 & 211 \\
3 & & & 68 & 286 & 218 \\
1 & & & 65 & 246 & 181 \\
2 & 180 & 1000 & 70 & 268 & 198 \\
3 & & & 66 & 274 & 208 \\
1 & & & 58 & 248 & 190 \\
2 & \multirow{2}{*}{200} & 59 & 54 & 251 & 192 \\
3 & & & 5900 & 257 & 203 \\
\hline
\end{tabular}

From the test results can be seen that the second test than the use of a test less time, indicating that the detection system in the detection of the same height of the beverage bottle products, combined with large data analysis of the detection system after the use of time than the manual detection of the same number of products Short, detection system with high efficiency.

\section{Conclusion}

Experimental results show that using machine vision system, combined with image data platform building, using machine learning feature recognition, the beverage bottle defect detection is more efficient. The system is more efficient and more stable under the condition of multi-sensor fusion. The system can effectively detect the defect of beverage bottle with high efficiency and high qualified rate, which meets the requirement of beverage bottle production line automation. Practical promotion of value, the market prospects.

\section{References}

[1] Xu Chi, Wang Zhiping, Ling Yongxiang, et al. Technology of beverage bottle defect detection based on intelligent vision system [J]. Automation of Instrumentation, 2011, 30 (1).

[2] Tong Jigang, Liao Fei, Luo Liangchuan, et al. An inspection system design for bottle and cans defect in machine vision [J] .Mechanical Engineering Technology, 2016,45 (8): 28-31.

[3] Liao Zilong, Zhong Shouyan. Measurement of Surface Mount Defects Based on Machine Vision Data Analysis [J]. Mechatronics Engineering Technology, 2016, 45 (8): 15-16, 45.

[4] Long Zhifan, Sun Zhihai, Kong Wanzeng et al. Reconfigurable Defect Detection of Industrial Vision Beverage Cap [J].

[5] Sun Tao. Based on image matching PET beverage bottle packaging defects detection study [D]. Guangdong University of Technology, 2008. 